\title{
DUALITY AND EILENBERG-MAC LANE SPECTRA
}

\author{
T. Y. LIN
}

\begin{abstract}
Stable cohomotopy groups of Eilenberg-Mac Lane spectra of finite groups are shown to be trivial. This implies that the stable homotopy category, which is large enough to represent ordinary cohomology theory, cannot be self-dual. It can also be interpreted as an evidence to support Freyd's generating hypothesis and a proof of a stable version of a conjecture of D. Sullivan.
\end{abstract}

\section{Introduction and statement of main results.}

1.1 Introduction. In this note we determine the stable cohomotopy groups $\pi^{*}(K(G))$ of Eilenberg-Mac Lane spectra $K(G)$ and the stable mapping groups $\{K(G), Y\}_{*}$ for a $\mathrm{CW}$-complex $Y$ of finite type. There are some interesting interpretations of this computation. The first is about SpanierWhitehead duality. The second is related to Freyd's generating hypothesis and a strong version of Barratt's conjecture (a weak version was solved recently in [10]). The third is related to a conjecture of D. Sullivan.

While this paper was in its final revision, the referee informed the author that the first theorem below can also be obtained from Margolis' result which appears in Proc. Amer. Math. Soc. 43 (1974), 409. Therefore we would like to offer the following remarks. From the statement of Margolis' result it appears that his result is more general than Theorem 3.2. However, from the remarks after Theorem 3.1 it is clear that our method can obtain Margolis' result also. Therefore it seems to this author that our method is an interesting, perhaps more general, alternative approach to his result. The backbone of our approach is to prove the edge isomorphism theorem without the information on the convergence of spectral sequences. This is quite useful because, in general, the convergence problem of a spectral sequence is harder than its construction. Finally the author would like to thank the referee for his valuable suggestions which brought this paper into the present form.

1.2 Statement of main results. We will state some of our results here. Let $\Sigma$ be Boardman's stable category and $\Sigma_{h}$ be the stable homotopy category. Let $K(G)$ be the Eilenberg-Mac Lane spectrum of an abelian group $G$ and $S^{0}$ be the 0 -sphere spectrum. Let $\{X, Y\}_{*}$ be the stable homotopy classes of maps of all degrees from $X$ to $Y$.

$$
\text { 3.2 THEOREM. }\left\{K\left(Z_{p}\right), S^{0}\right\}_{*}=0 \text {. }
$$

Received by the editors November 13, 1973 and, in revised form, March 10, 1975.

AMS (MOS) subject classifications (1970). Primary 55E45, 55E10.

Key words and phrases. Stable homotopy category, Spanier-Whitehead duality, Freyd's generating hypothesis, Eilenberg-Mac Lane spectrum, Moore spectrum, Adams spectral sequence, Steenrod algebra, inverse limit, dual of Milnor's Lemma. 
According to [7, Proposition 9.4] Freyd's generating hypothesis implies that there is no minimal ideal in the stable homotopy ring $\pi_{*}$ of spheres, or equivalently that $\operatorname{Hom}_{\pi_{*}}\left(Z_{p}, \pi_{*}\right)=0$. On the other hand, the strong version of Barratt's conjecture (i.e., the $p$-primary component of positive stems is a nilpotent ideal of $\pi_{*}$ ) implies the opposite. Hence, the proof or disproof of the conjecture $\operatorname{Hom}_{\pi_{*}}\left(Z_{p}, \pi_{*}\right)=0$ is quite an interesting problem. Although our result is inconclusive, nevertheless, it says that no element of $\operatorname{Hom}_{\pi_{*}}\left(Z_{p}, \pi_{*}\right)$ corresponds to a geometric map $K\left(Z_{p}\right) \rightarrow S^{0}$. Thus our result can be regarded as some evidence to support Freyd's generating hypothesis.

3.3 Corollary. Spanier-Whitehead duality cannot be extended to the homotopy category $\Sigma_{h}$.

In [5] Boardman has shown that a stable homotopy category cannot be selfdual if it allows the usual homotopy procedures. Our computations show, more specifically, that there is no dual object for Eilenberg-Mac Lane spectrum $K\left(Z_{p}\right)$. This is interesting because two major motivations for developing stable homotopy categories are Spanier-Whitehead duality and the Adams spectral sequence. Roughly, our results imply that if the stable homotopy category is extended so as to admit the Adams spectral sequence (which requires the existence of Eilenberg-Mac Lane spectra), then the extended category can no longer be self-dual.

3.4 COROLlaRY. $\left\{K\left(Z_{p}\right), X\right\}_{*}=0$ for any finite spectrum $X$. Or equivalently, any stable essential map from $K\left(Z_{p}\right)$ is incompressible.

D. Sullivan conjectured that there is no essential map from $R P^{\infty}=$ $K\left(Z_{2}, 1\right)$ to any finite $C W$-complex. Our results can be regarded as a stable version of Sullivan's conjecture.

\subsection{TheOREM.}

$$
\left\{K(Z), X^{0}\right\}_{*}= \begin{cases}\operatorname{Ext}_{Z}(Q, Z) & \text { if } *=-1 \\ 0 & \text { if } * \neq-1\end{cases}
$$

4.2 THEOREM. Let $Y$ be a $C W$-complex with finite skeleton in each dimension. Then $\{K(G), Y\}_{*}=\operatorname{Ext}_{Z}\left(G \otimes_{Z} Q, H_{*+1}(Y, Z)\right)$.

This theorem implies all the previous results. However, since they are the major motivations of this paper, the stress of the paper still will be on them.

In the whole paper we will not distinguish the notations between a spectrum and its homotopy type, a map and its stable homotopy class. $Z_{p}$ will mean the ring of integers $\bmod p$, and $H^{*}(X)$ will always mean $Z_{p}$ coefficients.

2. Reformulation of the Adams spectral sequence. In the classical formulation of the Adams spectral sequence, one requires that the second argument be finite dimensional. This condition is used to ensure the convergence. We will waive this condition here and describe an obstruction group for the convergence.

2.1 THEOREM (ASS). Let $Y$ be a spectrum with finite skeleton at each dimension, and $X$ be an arbitrary spectrum. Then there is a spectral sequence $E_{r}^{* * *}$ such that: 
(i) $E_{2}^{* * *}=\operatorname{Ext}_{\Theta}^{* * *}\left(H^{*}(Y), H^{*}(X)\right)$, where $\mathbb{Q}$ is the Steenrod algebra mod $p$.

(ii) $E_{\infty}^{*, *}$ is fitted into the short exact sequence $0 \rightarrow F^{s} / F^{s+1} \rightarrow E_{\infty}^{s, *} \rightarrow G^{s, *}$ $\rightarrow 0$ where the filtration $F^{s}$ of $\{X, Y\}_{*}$ and the bigraded group $G^{*, *}$ will be explained below. Moreover, the spectral sequence together with statements (i) and (ii) are natural with respect to $X$ and $Y$.

Let us recall that the ASS arises from the following Adams tower (see [1] for notations and construction)

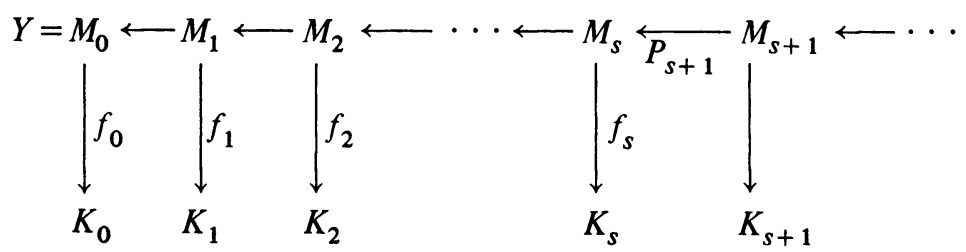

where $K_{i}$ are Eilenberg-Mac Lane objects and the sequences

$$
M_{s} \stackrel{f_{s}}{\longrightarrow} K_{s} \stackrel{j_{s}}{\longrightarrow} M_{s+1} \stackrel{P_{s+1}}{\longrightarrow} S M_{s} \approx M_{s}
$$

are exact triangles (note that $P_{s}$ is the composition of $q_{s}$ in [1] and desuspension). By applying the functor $\{X,-\}_{*}$ to the tower, we get an exact couple which gives the ASS. The filtration is defined by

$$
F^{s}=\operatorname{Im}\left[\left\{X, M_{s}\right\}_{*} \rightarrow\{X, Y\}_{*}\right]
$$

and the obstruction group $G^{* * *}$ is defined by

$$
G^{s, *}=\left(\operatorname{Ker} P_{s+1^{*}}\right) \cap\left(\underset{r \geqq 1}{\bigcap} \operatorname{Im}\left[\left\{X, M_{s+r}\right\}_{*} \rightarrow\left\{X, M_{s+1}\right\}_{*}\right]\right)
$$

where $P_{s^{*}}:\left\{X, M_{s}\right\}_{*} \rightarrow\left\{X, M_{s-1}\right\}_{*}$ is induced by $P_{s}: M_{s} \rightarrow M_{s-1}$.

Statement (i) is standard: statement (ii) follows from Eckman-Hilton's theory of exact couples (see [6, Theorem 4.16, p. 59]).

If the spectrum $X$ is finite dimensional, then one can show that $P_{s^{*}}$ are monomorphisms on

$$
I^{s}=\bigcap_{r} \operatorname{Im}\left[\left\{X, M_{s+r}\right\}_{*} \rightarrow\left\{X, M_{s}\right\}_{*}\right]
$$

and, hence, $G^{* *}=0$. This gives the usual convergent ASS.

Following the notation of $\S 5$, let us write $M_{\infty}=\varlimsup_{\leftarrow} M_{s}$.

2.2 Proposition. Let $Y$ be as in 2.1. Then if $H^{*}(Y)$ is free over the algebra generated by 1 and Bockstein operator, then $M_{\infty} \simeq *$.

Proof. From [1, §4, Remark 4 after Lemma 3], we get $\pi_{q}\left(M_{s}\right)=0$ for all sufficiently large $s$. Then by dual of Milnor's Lemma (Theorem 5.1), we have $\pi_{*}\left(M_{\infty}\right)=0$, i.e., $M_{\infty} \simeq *^{*}$.

This proposition can be strengthened if we use the notion of $E$-completion [3]. Let $\hat{L}_{p}$ be the Moore spectrum of $p$-adic integers $\hat{Z}_{p}$. Then $X \wedge \hat{L}_{p}$ is the $E$-completion of $X$, where $E$ is the ordinary homology theory with coefficient $Z_{p}$ and $X$ is a spectrum with finite skeletons at each dimension. Note that $H^{*}\left(X \wedge L_{p}\right)=H^{*}(X)$. Hence, by smashing $\hat{L}_{p}$ to the tower $\left(\begin{array}{c}* \\ *\end{array}\right)$ of $\S 2$, we 
still get the same ASS. Write $\hat{M}_{\infty}=\varlimsup_{s}\left(M_{s} \wedge \hat{L}_{p}\right)$. Then from the arguments in the proof of [3, Theorem 15.1(iii), $\stackrel{\leftarrow}{\text {. }}$ 250], we have the following:

\subsection{Proposition. Let $Y$ be as in 2.1. Then $\hat{M}_{\infty} \simeq$ * .}

3. Stable cohomotopy groups of Eilenberg-Mac Lane spectra. This section is the main body of the paper. Although the main theorems are special cases of $\S 4$, since they are the major motivation of this paper, we give complete proofs here. However, we have arranged the proofs in such a way that they can easily be adopted for general results in $\S 4$. In the proofs we have avoided the use of the notion of $E$-completion [3], however, it seems necessary for the results in $\$ 4$.

3.1. ThEOREM. $\left\{K\left(Z_{p}\right), L_{p}\right\}_{*}=0$, where $L_{p}$ is the Moore spectrum of $Z_{p}$.

Proof. Put $X=K\left(Z_{p}\right)$ into Theorem 2.1. Then, by self-injectiveness of $H^{*}\left(K\left(Z_{p}\right)\right)=\mathbb{Q}($ see [2], [9]), we have

$$
E_{2}^{s, *}=\operatorname{Ext}_{\mathscr{Q}}^{s, *}\left(H^{*}(Y), \mathbb{Q}\right)=0, \quad s>0 .
$$

Therefore, we have $E_{\infty}^{s, *}=0, s>0$. Moreover from Theorem 2.1, we have a one-to-one map (since $G^{s, *}=0$ )

$$
P_{s^{*}}: I^{s} \rightarrow I^{s-1}
$$

and

$$
0 \rightarrow F^{0} / F^{1} \rightarrow E_{\infty}^{0, *} \rightarrow 0
$$

and

$$
\left\{K\left(Z_{p}\right), Y\right\}_{*}=F^{0} \supset F^{1}=F^{2}=\cdots=F^{s}=\cdots .
$$

In other words, the length of the filtration on $\left\{K\left(Z_{p}\right), Y\right\}_{*}$ is $\leqq 2$. In particular, if we choose $Y=M_{s}$, where the $M_{s}$ are those spectra in Adams tower ( $\left.{ }_{\star}^{*}\right)$ of $\S 2$, the filtration on $\left\{K\left(Z_{p}\right), M_{s}\right\}_{*}$ is also of finite length. Hence, the following intersection is, in fact, a finite intersection.

$$
\begin{aligned}
I^{s} & =\bigcap_{r} \operatorname{Im}\left[\left\{K\left(Z_{p}\right), M_{s+r}\right\}_{*} \rightarrow\left\{K\left(Z_{p}\right), M_{s}\right\}_{*}\right] \\
& =\operatorname{Im}\left[\left\{K\left(Z_{p}\right), M_{s+1}\right\}_{*} \rightarrow\left\{K\left(Z_{p}\right), M_{s}\right\}_{*}\right] .
\end{aligned}
$$

Therefore the map $P_{s}$ induces an onto map

$$
P_{s^{*}}: I^{s} \rightarrow I^{s-1} \text {. }
$$

Combining (1) and (4), we have the following isomorphisms:

$$
I^{0} \cong I^{1}=I^{2} \cong \cdots \stackrel{P_{s}^{*}}{=} I^{s} \ldots .
$$

It is obvious from the definition of inverse limit that $\lim _{s}\left\{K\left(Z_{p}\right), M_{s}\right\}_{*}$ $=\lim _{\leftarrow} I^{s}$. Moreover, from the dual of Milnor's Lemma (see $\$ 5$ below),

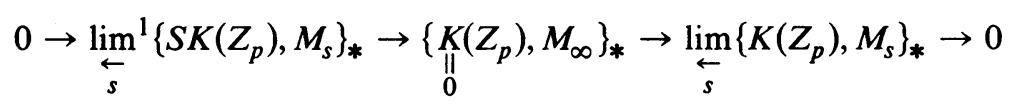


$\left(M_{\infty} \simeq *\right.$ by Proposition 2.2), we get

$$
0=\lim _{s}\left\{K\left(Z_{p}\right), M_{s}\right\}_{*}=I^{s} \text { for all } s .
$$

In particular, $I^{0}=0$. Then from (2) and (3), we have

$$
\left\{K\left(Z_{p}\right), Y\right\}_{*}=F^{0} \cong E_{\infty}^{0, *} \cong E_{2}^{0, *}
$$

i.e.

$$
\left\{K\left(Z_{p}\right), Y\right\}_{*}=\operatorname{Hom}_{\mathfrak{Q}}\left(H^{*}(Y), \mathfrak{Q}\right) .
$$

If we take $Y=L_{p}=S^{0} \cup_{p} e^{1}$ and note that $H^{*}\left(L_{p}\right)$ is an $Q$-module with nonvanishing groups only at degree 0 and 1 , we see immediately that there is no nontrivial $\mathbb{Q}$-map of $H^{*}\left(L_{p}\right) \rightarrow \mathbb{Q}$. That is,

$$
\operatorname{Hom}_{\mathscr{Q}}\left(H^{*}\left(L_{p}\right), \mathscr{Q}\right)=\left\{K\left(Z_{p}\right), L_{p}\right\}_{*}=0 .
$$

Therefore, we have the theorem.

REMARK. The isomorphism (5) proves a special case of Margolis' result (Proc. Amer. Math. Soc. 43 (1974), 409). For general $Y$, we proceed as follows: Using 2.3 to replace 2.2, we can proceed as in Theorem 3.1 to get

$$
\left\{K\left(Z_{p}\right), Y \wedge \hat{L}_{p}\right\}_{*} \cong \operatorname{Hom}_{\mathfrak{Q}}^{*}\left(H^{*}\left(Y \wedge \hat{L}_{p}\right), \mathbb{Q}\right) \cong \operatorname{Hom}_{\mathfrak{Q}}^{*}\left(H^{*}(Y), \mathfrak{Q}\right)
$$

So the only thing left to be proved is that $\left\{K\left(Z_{p}\right), Y \wedge \hat{L}_{p}\right\}_{*}=\left\{K\left(Z_{p}\right), Y\right\}_{*}$. For this let us consider the following commutative diagram:

$$
\begin{gathered}
\left\{K\left(Z_{p}\right), Y\right\}_{*} \stackrel{p^{t} \iota}{\longrightarrow}\left\{K\left(Z_{p}\right), Y\right\}_{*} \stackrel{i_{t}}{\longrightarrow}\left\{K\left(Z_{p}\right), Y \wedge L_{p^{t}}\right\}_{*} \rightarrow \\
\downarrow p \imath \quad \downarrow q_{t} \\
\left\{K\left(Z_{p}\right), Y\right\}_{*} \rightarrow\left\{K\left(Z_{p}\right), Y\right\}_{*} \stackrel{i_{t-1}}{\rightarrow}\left\{K\left(Z_{p}\right), Y \wedge L_{p^{t-1}}\right\}_{*} \rightarrow
\end{gathered}
$$

where $\iota$ is the identity map and $q_{t}$ is the map induced by the natural projection $Z_{p^{t}} \rightarrow Z_{p^{t-1}}$. Since each group is a $Z_{p}$ vector space, we have

$$
\begin{aligned}
& 0 \rightarrow\left\{K\left(Z_{p}\right), Y\right\}_{*} \stackrel{i_{i}}{\rightarrow}\left\{K\left(Z_{p}\right), Y \wedge L_{p^{t}}\right\}_{*} \rightarrow\left\{K\left(Z_{p}\right), Y\right\}_{*-1}-0 \\
& \downarrow=\quad \downarrow q_{t} \quad \downarrow 0 \\
& 0 \rightarrow\left\{K\left(Z_{p}\right), Y\right\}_{*} \stackrel{i_{t-1}}{\longrightarrow}\left\{K\left(Z_{p}\right), Y \wedge L_{p^{t-1}}\right\}_{*} \rightarrow\left\{K\left(Z_{p}\right), Y\right\}_{*-1}-0
\end{aligned}
$$

From this diagram one sees that the sequence of maps $q_{t}$ satisfies MittagLeffler condition (note that as $Z_{p}$-vector spaces the sequences above split and $q_{t}$ maps $\operatorname{Im} i_{t} \rightarrow \operatorname{Im} i_{t-1}$ isomorphically and the complement to zero). By the dual of Milnor's Lemma (5.1) we have

$$
\left\{K\left(Z_{p}\right), Y \wedge \hat{L}_{p}\right\}_{*}=\lim _{t}\left\{K\left(Z_{p}\right), Y \wedge L_{p^{t}}\right\}_{*} .
$$

Moreover the injections $i_{t}$ induce an isomorphism

$$
\left\{K\left(Z_{p}\right), Y\right\}_{*}=\lim _{\leftarrow}\left\{K\left(Z_{p}\right), Y \wedge L_{p^{\prime}}\right\}_{*}=\left\{K\left(Z_{p}\right), Y \wedge \hat{L}_{p}\right\}_{*} .
$$

This proves the remark. 
Next is our main theorem.

3.2 TheOREM. $\left\{K\left(Z_{p}\right), S^{0}\right\}_{*}=0$.

Proof. Note that $\left\{K\left(Z_{p}\right), S^{0}\right\}_{*}$ is a $Z_{p}$-vector space, therefore the Puppe sequence of $S^{0} \stackrel{p h}{\longrightarrow} S^{0} \rightarrow L_{p}$ reduces to

$$
\left.0 \rightarrow\left\{K\left(Z_{p}\right), S^{0}\right\}_{*} \rightarrow \underset{\substack{\|\|}}{K}\left(Z_{p}\right), L_{p}\right\}_{*} \rightarrow\left\{K\left(Z_{p}\right), S^{0}\right\}_{*} \rightarrow 0 .
$$

Now the main theorem follows immediately from Theorem 3.1 above.

3.3 Corollary. Spanier-Whitehead duality cannot be extended to the stable homotopy category $\Sigma_{h}$.

Proof. Let $D X$ be the dual of $X$. Then Spanier-Whitehead duality would give us

$$
\left\{K\left(Z_{p}\right), S^{0}\right\}_{*} \cong\left\{D S^{0}, D K\left(Z_{p}\right)\right\}_{*} .
$$

But $D S^{0}=S^{0}$ and, by Theorem 3.2, we have

$$
\pi_{*}\left(D K\left(Z_{p}\right)\right)=\left\{S^{0}, D K\left(Z_{p}\right)\right\}_{*}=\left\{K\left(Z_{p}\right), S^{0}\right\}_{*}=0 .
$$

That is, $D K\left(Z_{p}\right) \simeq *$. This shows that $D K\left(Z_{p}\right)$ cannot exist.

3.4 Corollary. $\left\{K\left(Z_{p}\right), X\right\}_{*}=0$ for any finite spectrum $X$.

Proof. Since $X$ can be obtained by attaching cells in a finite number of steps by using the Puppe sequence and 3.2 at each step, we have this corollary,

3.5 Corollary. $\left\{K(T), S^{0}\right\}_{*}=0$ for any torsion abelian group $T$.

Proof. First note that $T$ can be decomposed into a direct sum of its $p$ primary subgroups and $K(T)$ into the corresponding wedge. Thus one need only prove the case when $T$ is a $p$-primary torsion group. Let $T_{n}$ be the subgroup of elements $x$ in $T$ such that $p^{n} x=0$. Then we have an increasing sequence of groups. $T_{1} \subset T_{2} \subset \cdots \subset T_{n} \subset \cdots \subset T$ and a corresponding sequence for $K\left(T_{n}\right)$ 's. By Milnor's Lemma, the corollary is reduced to proving $\left\{K\left(T_{n}\right), S^{0}\right\}_{*}=0$. For this, we induct on $n$ by the exact triangles $K\left(T_{n}\right)$ $\rightarrow K\left(T_{n+1}\right) \rightarrow K\left(T_{n+1} / T_{n}\right)$ with the observations that $T_{n+1} / T_{n}$ and $T_{1}$ are direct sums of copies of $Z_{p}$.

\subsection{THEOREM.}

$$
\left\{K(Z), S^{0}\right\}_{*}= \begin{cases}\operatorname{Ext}_{Z}(Q, Z) & \text { if } *=1, \\ 0 & \text { if } * \neq-1 .\end{cases}
$$

Proof. Consider the sequence $K(Z) \rightarrow K(Q) \rightarrow K(Q / Z)$. Then the Puppe sequence and Corollary 3.5 give $\left\{K(Z), S^{0}\right\}_{*} \cong\left\{K(Q), S^{0}\right\}_{*}$. Note that $K(Q)$ is a Moore spectrum. Then by universal coefficient theorem [8, Chapter $5, \mathrm{p}$. 30] for stable homotopy groups, we have $\left\{K(Q), S^{0}\right\}_{-1}=\operatorname{Ext}_{Z}(Q, Z)$ and zero in all other degrees. This proves the theorem.

4. Further results. In this section, we extend our computations to more general situations. Recall that a CW-complex can be regarded as an object in 
$\Sigma$, hence we can consider the stable maps from a spectrum to a $\mathrm{CW}$-complex. First we prove

4.1 Theorem. Let $Y$ be a $C W$-complex. Then $\operatorname{Hom}_{\mathscr{Q}}\left(H^{*}(Y), \mathbb{Q}\right)=0$.

Proof. Let $y \in H^{*}(Y)$. Then from [11, pp. 1 and 76], $P^{i} y=0$ if $2 i$ $>\operatorname{dim} y$ [or, $\mathrm{Sq}^{i} y=0$ if $i>\operatorname{dim} y$ ]. On the other hand, for any $a \in \mathbb{Q}, P^{i} a \neq 0$ [or $\mathrm{Sq}^{i} a \neq 0$ ] for sufficiently large $i$. Therefore there are no nontrivial $\mathbb{Q}$-maps from $H^{*}(Y)$ to $\mathbb{Q}$.

4.2 Theorem. Let $Y$ be a $C W$-complex with finite skeleton at each dimension, and $G$ be an abelian group. Then

$$
\{K(G), Y\}_{*}=\operatorname{Ext}_{Z}\left(G \otimes_{Z} Q, H_{*+1}(Y, Z)\right) .
$$

Proof. (1) From 4.1 and the remark of 3.1, we have $\left\{K\left(Z_{p}\right), Y\right\}_{*}=0$.

(2) Following the pattern of arguments in 3.5, we have $\{K(T), Y\}_{*}=0$ for any torsion group $T$.

(3) Let $T$ be the torsion subgroup of $G$. Then $G / T$ is a torsion free abelian group, and hence we have an exact triangle

$$
K\left(G / T \otimes_{Z} Z\right) \rightarrow K\left(G / T \otimes_{Z} Q\right) \rightarrow K\left(G / T \otimes_{Z} Q / Z\right) .
$$

By the result in (2), we have $\{K(G / T), Y\}_{*} \cong\left\{K\left(G / T \otimes_{Z} Q\right), Y\right\}_{*}$. Observe that $G / T \otimes_{Z} Q=G \otimes_{Z} Q$, so we have

$$
\{K(G), Y\}_{*} \simeq\{K(G / T), Y\}_{*}=\left\{K\left(G \otimes_{Z} Q\right), Y\right\}_{*} .
$$

(4) Next note that $K\left(G \otimes_{Z} Q\right)$ is a Moore spectrum; hence by universal coefficient theorem for homotopy groups we have

$$
\left\{K\left(G \otimes_{Z} Q\right), Y\right\}_{q} \cong \operatorname{Ext}_{Z}\left(G \otimes_{Z} Q, \pi_{q+1}(Y)\right) .
$$

From Hurewicz isomorphism theorem, we see that the kernel and cokernel of the Hurewicz homomorphism $\pi_{*}(Y) \rightarrow H_{*}(Y ; Z)$ are finitely generated torsion (= finite) groups. Since $\operatorname{Ext}_{Z}\left(G \otimes_{Z} Q, T\right)=0$ for any finite group $T$, we have

$$
\operatorname{Ext}_{Z}\left(G \otimes_{Z} Q, \pi_{q+1}(Y)\right)=\operatorname{Ext}_{Z}\left(G \otimes_{Z} Q, H_{q+1}(Y ; Z)\right) .
$$

Thus we have

$$
\{K(G), Y\}_{*}=\left\{K\left(G \otimes_{Z} Q\right), Y\right\}_{*}=\operatorname{Ext}_{Z}\left(G \otimes_{Z} Q, H_{*+1}(Y ; Z)\right) .
$$

This proves the theorem.

5. Dual of Milnor's Lemma. In $\Sigma$ the direct product may not exist, hence the inverse limit in the strict sense usually does not exist. In this note, we will construct "homotopy inverse limits" which will be suitable for our purpose.

5.1 THEOREM. For a given sequence of spectra in $\Sigma$,

$$
\rightarrow X_{n} \stackrel{f_{n}}{\longrightarrow} X_{n-1} \rightarrow \cdots \rightarrow X_{1} \stackrel{f_{1}}{\rightarrow} X_{0} .
$$

We construct a spectrum $X_{\infty}$ with maps $q_{n}: X_{\infty} \rightarrow X_{n}$ such that $q_{n-1} \simeq f_{n} \cdot q_{n}$ 
and a short exact sequence

$$
0 \rightarrow \lim _{\leftarrow}^{1}\left\{S Y, X_{n}\right\}_{*} \rightarrow\left\{Y, X_{\infty}\right\}_{*} \rightarrow \lim _{\leftarrow}\left\{Y, X_{n}\right\}_{*} \rightarrow 0
$$

natural in $Y$.

The spectrum $X_{\lim }$ will be called the homotopy inverse $\operatorname{limit}, \overline{\lim } X_{n}$, of the sequence, and the short exact sequence will be called the dua ' of Milnor's Lemma.

Proof. Let $\prod_{n=0}^{\infty} X_{n}, \prod_{n=1}^{\infty} X_{n}$ be the direct products. According to [4, C.22] they exist up to homotopy types. Let

$$
f=\prod_{n=1}^{\infty} f_{n}: \prod_{n=1}^{\infty} X_{n} \rightarrow \prod_{n=0}^{\infty} X_{n}
$$

be the product of maps; it also exists only up to homotopy type.

Let $\bar{f}$ be the composition

$$
\bar{f}: \prod_{n=0}^{\infty} X_{n} \stackrel{q}{\rightarrow} \prod_{n=1}^{\infty} X_{n} \stackrel{f}{\rightarrow} \prod_{n=0}^{\infty} X_{n}
$$

where $q$ is the natural projection. Let $X$ be the mapping cone of the map $1-\bar{f}$, which is the difference of identity and $\bar{f}$. Then the Puppe sequence of the exact triangle

$$
\prod_{n=0}^{\infty} X_{n} \stackrel{1-\bar{f}}{\longrightarrow} \prod_{n=0}^{\infty} \rightarrow X_{\infty} \stackrel{\delta}{\longrightarrow} \prod_{n=0}^{\infty} X_{n}
$$

gives

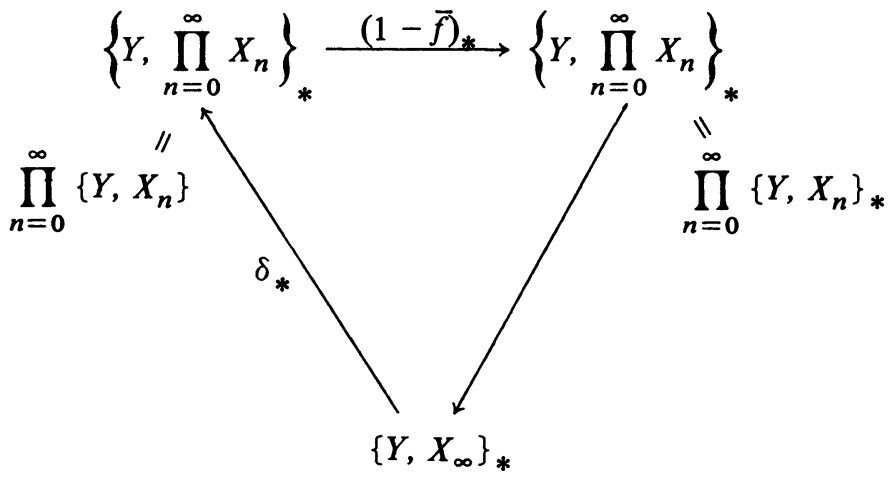

Then from the definitions of $\lim _{\leftarrow}$ and $\lim ^{1}$, we get

$$
0 \rightarrow \lim _{\leftarrow}^{1}\left\{S Y, X_{n}\right\}_{*} \rightarrow\left\{Y, X_{\infty}\right\}_{*} \rightarrow \lim _{\leftarrow}\left\{Y, X_{n}\right\}_{*} \rightarrow 0 .
$$

Next we need to construct $q_{n}: X_{\infty} \rightarrow X_{n}$. Let $p_{n}: \prod_{n=0}^{\infty} X_{n} \rightarrow X_{n}$ be the projection and write $q_{n}=p_{n} \cdot \delta$ where $\delta$ is the coboundary operator of (6) above, we have $p_{n} \cdot \bar{f} \simeq f_{n+1} \cdot p_{n+1}$ and $(1-\bar{f}) \delta \simeq *$. Thus we have

$$
q_{n} \equiv p_{n} \cdot \delta \simeq p_{n} \cdot \bar{f} \cdot \delta \simeq f_{n+1} \cdot p_{n+1} \cdot \delta \equiv f_{n+1} \cdot q_{n+1} .
$$

This shows that the $q_{n}$ are the desired maps. Thus we have proved the theorem. 
Remark. If $X_{n}$ had arisen from the Postnikov tower of some spectrum, say $Y$, then $X_{\infty} \simeq Y$. This follows immediately from the dual of Milnor's Lemma and the fact that $\lim ^{1} \pi_{*}\left(X_{n}\right)=0$.

\section{REFERENCES}

1. J. F. Adams, Stable homotopy theory, Springer-Verlag, Berlin and New York, 1964. MR 32 \#3061.

2. J. F. Adams and H. R. Margolis, Modules over the Steenrod algebra, Topology 10 (1971), 271-282. MR 45 \#3520.

3. J. F. Adams, Stable homotopy and generalized homology, Mathematics Lecture Notes, Univ. of Chicago, 1971.

4. J. Boardman, Stable homotopy theory, mimeograph notes, Univ. of Warwick, Coventry, England, 1965.

5. _- Stable homotopy theory is not self-dual, Proc. Amer. Math. Soc. 26 (1970), 369-370. MR 42 \#3784.

6. B. Eckmann and P J. Hilton, Exact couples in an abelian category, J. Algebra 3 (1966), 38-87. MR 33 \# 164.

7. P. Freyd, Stable homotopy, Proc. Conf. Categorical Algebra (La Jolla, Calif., 1965), Springer, New York, 1965, pp. 121-172. MR 35 \#2280.

8. P. J. Hilton, Homotopy theory and duality, Gordon and Breach, New York and London, 1965. MR 33 \#6624.

9. J. Moore and F. Peterson, Nearly Frobenius algebra, Poincaré algebra and their modules, J. Pure Appl. Algebra 3 (1973), 83-93.

10. G. Nishida, The nilpotent of elements of the stable homotopy groups of spheres, 1973 (preprint).

11. N. E. Steenrod and D. B. A. Epstein, Cohomology operations, Ann. of Math. Studies, no. 50, Princeton Univ. Press, Princeton, N. J., 1962. MR 26 \# 3056.

Department of Mathematics, Purdue University, West Lafayette, Indiana 47907

Current address: Department of Mathematics, University of South Carolina, Aiken, South Carolina 29801 